\title{
Prediction of Mortality and Clinical Decisions Using Frailty Scores in Hospitalized Elderly with Covid-19
}

\author{
Jorg MP Franssen, $M D^{1}$, Robert $M$ Kwee, $M D$, PhD', Martijn D de Kruif, $M D$, $P h D^{3}$, Yvette \\ AW Sipers, $M s c^{1}$, Pathman Pasupathy, $M D^{3}$, Frederik Plum, $M D^{1}$ and Walther MWH Sipers, \\ $M D, \mathrm{PhD}^{1^{*}}$ (iD
}

${ }^{1}$ Department of Geriatric Medicine, Zuyderland Medical Center, Heerlen/Sittard/Geleen, The Netherlands

${ }^{2}$ Department of Radiology, Zuyderland Medical Center, Heerlen/Sittard/Geleen, The Netherlands

${ }^{3}$ Department of Pulmonology, Zuyderland Medical Center, Heerlen/Sittard/Geleen, The Netherlands

\begin{abstract}
Aim: Many decisions in clinical care in elderly patients admitted to the hospital with COVID-19 are based upon mortality risk. Screening for frailty is recommended, however its predictive value in hospitalized older adults with COVID-19 infection is unknown.

Objective is to compare the prognostic power of frailty scores with other prognostic parameters including age, laboratory biomarkers, comorbidities and imaging findings. In addition, to determine usefulness of clinical frailty scores in clinical decision-making including instalment of Do-Not-Intubate (DNI) order and discharge to institutionalized care.

Methods: A single-centre retrospective observational study in hospitalized patients $>65$ years with COVID-19. Clinical Frailty Scale (CFS) and/or Groningen Frailty Indicator (GFI), Charlson Comorbidity Index (CCI), inflammation, CT severity scores (Do-Not-Intubate) DNI order, in hospital mortality and discharge destination were determined using chart data as well as data from the ZuydErLand COVID-19 regiStry (ELVIS).

Results: 157 patients were included, mean age $77 \pm 7$ years. $30 \%$ was considered frail. The frailty scores failed to predict mortality. However, the frailty scores appeared to be associated with clinical decision-making including instalment of DNI orders and discharge to institutionalized care.
\end{abstract}

Conclusions: Clinical frailty scores in our study showed promise as a tool for decision making in COVID-19, but probably not for prediction of mortality.

\section{Keywords}

Clinical Frailty Scale, COVID-19, Frailty, Groningen Frailty Indicator, Mortality

\section{Introduction}

COVID-19 is the disease caused by Severe Acute Respiratory Syndrome CoronaVirus 2 (SARS-CoV-2) [1]. COVID-19 is more lethal in older patients. Multiple recent studies stated that age is a highly predictive measure of severe COVID-19 disease [1-5].

Frailty is known to be a predictor of multiple adverse outcomes such as mortality, institutionalisation and functional decline [6]. Frailty is characterized by widespread physiological declines across many systems that can leave a person more susceptible to health stressors, such as COVID-19 infection [7]. Screening for frailty in hospitalized older adults because of COVID-19 infection with a risk of respiratory failure seems therefore mandatory. Frailty could be a more important predictor of outcome than age [8]. Therefore the National Institute for Health and Care Excellence (NICE) in the Unit- ed Kingdom included the Clinical Frailty Scale (CFS) in their COVID-19 rapid guideline to determine the frailty of patients who are 65 years or older [9].

The CFS is generally accepted as a valid tool to determine and classify frailty [10]. In our teaching hospital, screening

*Corresponding author: Walther MWH Sipers, MD, PhD, Department of Geriatric Medicine, Zuyderland Medical Center, dr. H. van der Hoffplein 1, 6162 BG Geleen, The Netherlands, Tel: 0031884597777

Accepted: February 20, 2021

Published online: February 22, 2021

Citation: Franssen JMP, Kwee RM, Kruif MD, et al. (2021) Prediction of Mortality and Clinical Decisions Using Frailty Scores in Hospitalized Elderly with Covid-19. T Geriatr Med 3(1):49-54 
for frailty has been performed for years using the Groningen Frailty Indicator (GFI) at hospital admission [11].

There is lack of knowledge about the predictive value of the CFS and the GFI of targeting therapy and of adverse outcome(s) in hospitalized older adults with COVID-19 infection. Therefore we aimed to determine the prognostic properties of the CFS and GFI for prediction of mortality. Furthermore, we aimed to compare the prognostic power with other prognostic parameters including age, laboratory biomarkers, comorbidities and imaging findings. In addition, we wished to determine usefulness of clinical frailty scores in clinical decision making including instalment of Do-Not-Intubate (DNI) order and discharge to institutionalized care.

\section{Methods}

\section{Study sample}

A single-centre retrospective observational study in patients who are 65 years and older, hospitalized because of COVID-19 in Zuyderland medical centre was conducted between 26 March 2020 until 26 April 2020.

Inclusion criteria were COVID-19 Polymerase Chain Reaction (PCR) positive or high clinical suspicion and a CFS at hospitalization. Diagnosis of COVID-19 infection was based on a combination of typical patient history, laboratory markers, desaturation findings, a positive PCR test and a CO-RADS score. CO-RADS score is based on the CT findings, the level of suspicion of COVID-19 infection is graded from very low or CO-RADS 1 up to very high or CO-RADS 5 [12]. In case of doubt and a negative PCR result, cases were discussed by the clinical team until agreement was reached for high or low suspicion.

A physician assistant, a research nurse and a resident of the geriatric department gathered patient data from the electronic medical hospital file and completed the research database, together with data from the ZuydErLand COVID-19 regiStry (ELVIS). Data included; age, gender, diagnosis at hospital admission, medical history and laboratory data and outcome data. The data collection process was supervised by two staff members of the geriatric department (WS and FP) who controlled the data independently.

This study complied with the guidelines set out in the Declaration of Helsinki and was approved by the Medical Ethics Committee Zuyd, the Netherlands (METCZ20200079).

\section{Clinical frailty scale}

CFS is generally accepted as a valid tool to determine and classify frailty [10]. It depicts the physical functional impairment and ranges from 1 (very fit) to 9 (terminally ill). On the day the patient was attended to the emergency department the nurses of the geriatric department gathered information by making calls with patients, their family and general practitioner and used all the information in the electronic patient file to complete a CFS score in electronic patient file.

In our study, the CFS was divided into two categories of patients; non-frail patients with a CFS score with a cut-off point of $<4$ and frail patients with a CFS score $\geq 4$ [10].

\section{Groningen frailty indicator}

The Groningen Frailty Indicator (GFI) is a multi-domain frailty instrument, including physical, functional performance and cognition which ranges from 0 to 15: A score of 4 or higher indicates frailty [11]. The GFI is automatically generated due to the standard questions in the anamnesis and a checkbox in the electronic patient file by the attending hospital ward nurses. Subsequently, scores were divided into two categories: non-frail with a GFI score of 1-3 and frail with a GFI score of $\geq 4[11]$.

\section{Comorbidity and COVID-19 disease severity}

Charlson comorbidity index: $\mathrm{CCl}$ is a validated tool to predict 10-year survival in patients with multiple comorbidities and is used to categorize the comorbidities [13]. CCl was also used to categorize the comorbidities into pulmonary disease, cancer, diabetes mellitus, cognitive disorders and cardiovascular disease [13]. The $\mathrm{CCl}$ was assessed retrospectively by a resident of the geriatric department of the based on the information in the electronic medical file of the patient.

Chest CT severity score: Chest CT was a standard procedure upon emergency department admission for patients with suspected COVID-19 during the COVID-19 peak in our region. Chest CT was performed on either a 64-slice CT scanner (Philips Incisive) or on a 64-slice dual source scanner (Siemens Somatom Definition Flash).

The chest CT severity score was assessed to determine the extent of lung involvement. Chest CT scans were retrospectively evaluated by a radiologist (RK) who was blinded to all clinical information. The lungs were divided into 6 zones: The upper zones (above the carina), the middle zones (below the carina up to the inferior pulmonary vein), and the lower zones (below the inferior pulmonary vein) [14]. A semi-quantitative score was assigned to each lung zone: 0 ( $0 \%$ involvement), 1 (<25\% involvement), 2 (25-49\% involvement), 3 (50-74\% involvement), or 4 (> 75\% involvement) [14]. The individual scores of all lung zones were added, which resulted in a total lung score ranging from 0 to 24 [14].

\section{Statistics}

SPSS version 26 was used. Patients' characteristics were described by mean \pm SD and range for continuous variables and by frequencies and percentages for the categorical variables. Independent T-tests and Chi-square tests were used (normal distributed) to test the differences between groups.

Multivariate Logistic Regression was used to determine the impact of frailty, age and comorbidity ( $\mathrm{CCl}$ score) upon mortality and discharge to an institution.

\section{Results}

\section{Patient characteristics}

During the study period, 604 patients $>65$ years were admitted to the hospital. After exclusion of patients without proven or highly suspected COVID-19 $(n=313)$ and without a completed frailty score $(n=134), 157$ elderly patients with COVID-19 who were admitted to the hospital, were included 
Citation: Franssen JMP, Kwee RM, Kruif MD, et al. (2021) Prediction of Mortality and Clinical Decisions Using Frailty Scores in Hospitalized Elderly with Covid-19. T Geriatr Med 3(1):49-54

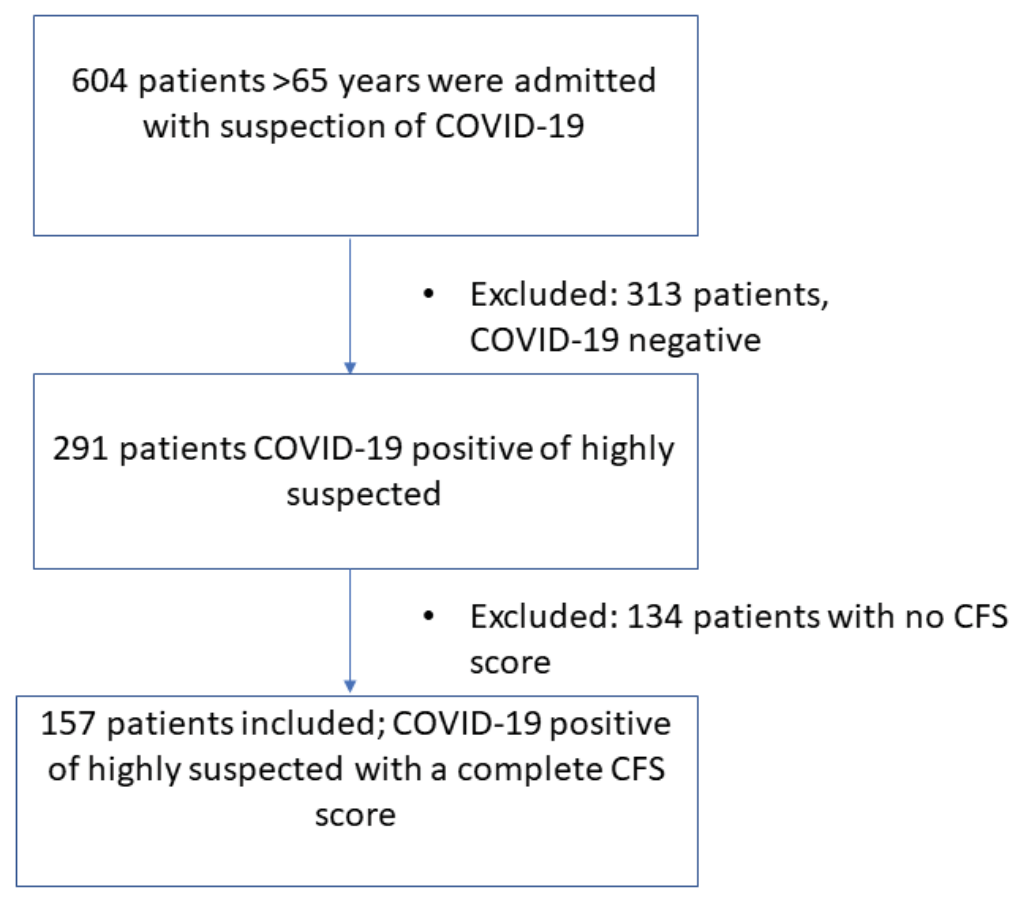

Figure 1: Flowchart inclusion.

Table 1: Baseline patient characteristics of patients aged 65-y and older hospitalized with a COVID-19 infection $(n=157)$.

\begin{tabular}{|l|l|}
\hline & Patients (\%) \\
\hline Age (years) & $77 \pm 7$ \\
\hline Gender (male) & $100(64 \%)$ \\
\hline COVID-19 PCR proven & $124(79 \%)$ \\
\hline Charlson Comorbidity Index, points (mean + sd) & $6.3 \pm 2.6$ \\
\hline Chronic Obstructive Pulmonary Disease & $41(26 \%)$ \\
\hline Cardiovasculair disease & $92(59 \%)$ \\
\hline Diabetes mellitus & $38(24 \%)$ \\
\hline Cognitive disorders & $24(15 \%)$ \\
\hline CT-severity score, points (mean + sd) & $7.5 \pm 6.2$ \\
\hline C-reactive protein at admission (mg/l) & $143 \pm 111$ \\
\hline
\end{tabular}

in the study (Figure 1). A combination of both CFS and GFI was completed for 145 patients. Patients characteristics are presented in (Table 1). The mean age of the patients was $77 \pm$ $7 y$ and 100 (64\%) patients were male.

\section{Frailty scores and mortality}

The mean CFS score was $3.0 \pm 1.5$ in 157 patients and the mean GFI score was $2.9 \pm 2.3$. In-hospital mortality was $30 \%$ $(n=47)$. The mean CFS and GFI scores were not significantly different between survivors and non-survivors (Table 2). Also in a multiple logistic regression model using correction for age, CT-severity score, $\mathrm{CCl}$ and $\mathrm{CRP}$, both frailty scores did not predict mortality (Table 3 ).

\section{Other predictors of mortality}

Age was not different between survivors and non-sur-

Table 2: Patient characteristics differences in survivors versus non-survivors, in older hospitalized patients with COVID-19 infection ( $n=157)$.

\begin{tabular}{|l|l|l|l|}
\hline & $\begin{array}{l}\text { Survivors } \\
\text { mean } \pm \mathrm{sd} \\
\mathrm{n}=110\end{array}$ & $\begin{array}{l}\text { Non-survivors } \\
\text { mean }+\mathrm{sd} \\
\mathrm{n}=47\end{array}$ \\
\hline Age, years & $76 \pm 7$ & $78 \pm 7$ & n.s \\
\hline CFS, points & $3.0 \pm 1.4$ & $3.1 \pm 1.9$ & n.s \\
\hline GFI, points & $2.8 \pm 2.4$ & $3.2 \pm 2.0$ & n.s \\
\hline CCI, points & $6.0 \pm 2.7$ & $6.8 \pm 2.4$ & P = 0.072 \\
\hline CT-severity, points & $6.5 \pm 5.2$ & $9.7 \pm 7.5$ & $181 \pm 127$ \\
\hline CRP, mg/I & $127 \pm 100$ & $P=0.009$ & $P=0.004$ \\
\hline
\end{tabular}

CFS: Clinical Frailty Scale; GFI: Groningen Frailty Indicator; CCI: Charlson Comorbidity Index; n.s: not significant 
Citation: Franssen JMP, Kwee RM, Kruif MD, et al. (2021) Prediction of Mortality and Clinical Decisions Using Frailty Scores in Hospitalized Elderly with Covid-19. T Geriatr Med 3(1):49-54

Table 3: Independent predictors of in-hospital mortality, in older hospitalized patients with COVID-19 infection.

\begin{tabular}{|l|l|l|}
\hline & $\begin{array}{l}\text { Odds ratio } \\
\text { in-hospital mortality (95\% Confidence interval) }\end{array}$ & Significance \\
\hline Clinical Frailty Scale & $1.28(0.51-3.23)$ & n.s \\
\hline Groningen Frailty Indicator & $1.76(0.67-4.59)$ & n.s \\
\hline Age & $1.01(0.95-1.08)$ & n.s \\
\hline Charlson Comorbidity index & $1.27(1.06-1.51)$ & $\mathrm{P}=0.010$ \\
\hline CT severity score & $1.13(1.05-1.22)$ & $\mathrm{P}=0.001$ \\
\hline C-reactive protein & $1.01(1.00-1.01)$ & $\mathrm{P}=0.038$ \\
\hline
\end{tabular}

n.s: not significant

Table 4: Patient characteristics of discharge destination and the independent predictors of institutionalization of older hospitalized patients with COVID-19 infection.

\begin{tabular}{|l|l|l|l|l|l|}
\hline & $\begin{array}{l}\text { Institutionalized } \\
\text { mean + sd } \\
\mathrm{n}=37\end{array}$ & $\begin{array}{l}\text { Home after } \\
\text { hospitalisation } \\
\text { mean + sd } \\
\mathrm{n}=79\end{array}$ & $\begin{array}{l}\text { Significance } \\
\text { (2-tailed) }\end{array}$ & $\begin{array}{l}\text { Odds ratio } \\
\text { Institutionalization } \\
\text { (95\% Confidence interval) }\end{array}$ & Significance \\
\hline CFS, points & $3.4 \pm 1.7$ & $2.7 \pm 1.2$ & $\mathrm{P}=0.022$ & $1.78(0.65-4.90)$ & $\mathrm{n} . \mathrm{s}$ \\
\hline GFI, points & $4.4 \pm 3.0$ & $2.1 \pm 1.8$ & $\mathrm{P}<0.001$ & $3.76(1.43-9.90)$ & $\mathrm{P}=0.007$ \\
\hline Age, years & $81 \pm 8$ & $75 \pm 6$ & $\mathrm{P}=0.001$ & $1.09(1.02-1.16)$ & $\mathrm{P}=0.017$ \\
\hline CCI, points & $7.2 \pm 2.9$ & $5.7 \pm 2.6$ & $\mathrm{P}=0.005$ & $1.08(0.90-1.31)$ & $\mathrm{n} . \mathrm{S}$ \\
\hline $\begin{array}{l}\text { CT-severity, } \\
\text { points }\end{array}$ & $5.6 \pm 5.8$ & $7.2 \pm 4.9$ & $\mathrm{n} . \mathrm{s}$ & $1.02(0.93-1.12)$ & $\mathrm{n}$ \\
\hline
\end{tabular}

CFS: Clinical Frailty Scale; GFI: Groningen Frailty Indicator; CCI: Charlson Comorbidity Index; n.s: not significant

vivors (Table 2). In non-survivors, significantly increased CT severity scores and levels of CRP ( $p=0.009$ and $p=0.004$, respectively) and a trend to significant $\mathrm{CCl}$ scores $(\mathrm{p}=0.072)$ were found. Multiple regression analysis showed that $\mathrm{CCl}$ scores, CT severity scores and levels of CRP all were independent predictors of mortality in this patient group (Table 3). Of note, the CT-severity scores were significantly higher in the non-survivors who were not frail in comparison to frail non-survivors (mean $11.5 \pm 7.2$ vs. $6.3 \pm 7.1 ; p=0.02$ ).

\section{Frailty and Do-Not-Intubate order}

COVID-19 could lead to respiratory failure and the need of mechanical ventilation. Especially in frail older adults the harm often does not outweigh the benefits of mechanical ventilation. In order to validate the frailty scores in COVID-19 in the elderly, the scores were compared between patients who did or did not receive a Do-Not-Intubate (DNI) order by the treating clinician. A DNI was issued for 112 (71\%) patients. Patients who were later categorized into being frail, had almost all received a DNI order: (CFS: $100 \%$ (47/47 patients) respectively GFI: 40/43). This was significantly different from patients who were categorized as non-frail (CFS: 65/110 respectively GFI: 64/102) ( $p<0.001)$.

\section{Factors predicting discharge destination}

Fifty percent of all the patients were sent home after hospital admission. Patients discharged to an institution compared to those who discharged to home, were significant more frail (Table 4). Multiple regression analysis revealed that the GFI and age independently predicted discharge to an institute (Table 4). CFS and CCI were not independent predictors of institutionalization.

\section{Discussion}

In this study in elderly patients admitted to the hospital with COVID-19, the CFS and GFI frailty scores failed to predict mortality. Also age did not influence survival. However, mortality was significantly predicted by other factors including increased levels of CRP, CT severity scores and the number of comorbidities expressed by $\mathrm{CCl}$. Another important finding in our study is that in contrast to the negative results for prediction of mortality, the GFI appeared to be highly useful for predicting need for institutional revalidation after discharge, whereas this could not be predicted by CFS, levels of CRP, CT severity scores and $\mathrm{CCl}$.

The negative findings in our study contradict previous literature in other diseases including pneumonia [15]. A possible explanation for the absence of this relation could be the absence of really frail older patients in our population. The absence of these patients in our hospital is probably the result of an agreement made between general practitioners, nursing homes and hospital not to refer very frail elderly patients, e.g. those who live in nursing homes for hospital admission. A recent Belgium study of 81 patients, $52 \%$ were long-term care residents, with a mean age of 85 and a median CFS of 7 points demonstrated that frailty was weakly associated with in-hospital mortality, however the population in this study was evidently more frail [16]. Another explanation may be 
that previous studies [16,17] applied a cut-off value for frailty at $\geq 5$, whereas in our study a cut-off of $\geq 4$ was applied. However, this is in agreement with earlier research by Rockwood, et al., they pointed out that the prognoses clearly decline at $\geq$ 4 points on the CFS [10].

Otherwise it is possible that patients were overestimated in the clinical frailty score assessment and therefore were categorized as non-frail with a CFS score of 3 points instead of 4. Those 'overestimated' patients could minimalize the difference in in-hospital mortality between the frail and non-frail patients. Finally, the lack of prognostic power of the frailty scores in our study might be explained due to difference in disease severity of COVID-19 in the non-frail compared to frail older patients. The CT-severity score in the non-frail deceased patients was significant higher compared to the deceased frail patients $(11.5 \pm 7.2$ vs. $6.3 \pm 7.1, p=0.02)$.

Frailty scores in our study were associated with clinical decisions regarding DNI orders. All the frail patients according the CFS and 93\% according the GFI had a DNI order compared to the fit patients in respectively $65 \%$ and $63 \%$. These results are supported by others. In a large population based cohort study in New Zealand with frail patients admitted to the ICU aged 80 years and older in a non-COVID-19 period had significantly poorer health outcomes than the non-frail patients [18].

Although CFS and GFI assess different domains of function, almost all patients classified as frail by both CFS or GFI had a DNI order. This could be due to the fact that age, comorbidities and the choice of the patient were also contributing factors in receiving a DNI order. A previous study of 502 patients with a mean age of 76 years underline that comorbidities are an important predictor of a DNI or ICU treatment agreement [19].

Therefore we suggest, based on our data and earlier research that a DNI order is an appropriate medical decision to prevent an unnecessary suffering with an expected bad outcome in the frail older patients with COVID-19 infection.

The mortality rate in our study is $29.9 \%$, which is higher compared to previous findings. In a review of the available data of the previous COVID-19 studies so far, the mortality rate is about $13.9 \%-21 \%$ in hospitalized patients regardless of age $[20,21]$. A recent study of hospitalized older adults with COVID-19 showed a mortality rate of $24 \%$, however there was no information about comorbidities in their population besides dementia [16]. Our higher mortality rate could be explained because our study population included only older patients with more comorbidities $(\mathrm{CCl}$ was 6.3 in our study versus 4.0 in a large cohort of 5700 patients with a mean age of 63-years-old) [21]. The presence of comorbidities was a predictor of adverse outcome in our study, these results were in line with previous findings [21,22].

Additionally, no information was available about complications, such as thrombo-embolic events, which could have elevated the mortality rate.

Interestingly, frailty scores in our study were related to a higher risk of not discharging home. These results are sup- ported by previous findings $[23,24]$. Frail patients are especially at risk for functional decline with loss of mobility and increased care dependency with a high risk of going to an institution, like a nursing home or rehabilitation unit [25].

Additionally, only GFI is an independent predictor of not discharging home, in contrary to the CFS. This difference could be explained because the GFI assesses multiple domains of frailty including cognition and mood [11] whereas CFS mainly asses frailty of the functional domain [10]. Both screening tools can be assessed in a short period of time and are easy to perform.

A limitation could be the missing of some patients because of lacking the CFS score. This was because the enormous amount of patients came to our hospital and there weren't all the resources available to determine the CFS in all our patients. Twelve patients were missed in the GFI screening. These patients were admitted directly to ICU or were hospitalized with an immediately installed palliative care due to severe illness. Those patients could have an elevated GFI score, so the mean GFI could have been higher in our study.

Concluding, our study demonstrated no predictive value of the frailty scores CFS and GFI for prediction of mortality in elderly patients with COVID-19. Because there are many possible explanations, larger multicentre studies can possible give the answer on this issue. Despite this outcome, the frailty scores appeared to be associated with clinical decision-making including instalment of DNI orders and discharge to institutionalized care. However only the GFI was an independent predictor of discharge to an institution. As such, clinical frailty scores in our study showed promise as a tool for decision making in COVID-19, but probably not for prediction of mortality.

\section{Acknowledgements}

Nothing to declare.

\section{Disclosure Statement}

This research did not receive any funding from agencies in the public, commercial, or not-for-profit sectors. The authors report no conflict of interest or competing interests.

\section{References}

1. Team N (2020) Vital surveillances: The epidemiological characteristics of an outbreak of 2019 novel coronavirus diseases (COVID-19) - China. China CDC Weekly 2: 113-122.

2. Hou W, Zhang W, Jin R, et al. (2020) Risk factors for disease progression in hospitalized patients with COVID-19: A retrospective cohort study. Infect Dis 52: 498-505.

3. Yang X, Yu Y, Xu J, et al. (2020) Clinical course and outcomes of critically ill patients with SARS-CoV-2 pneumonia in Wuhan, China: A single-centered, retrospective, observational study. Lancet Respir Med 8: 475-481.

4. Wu Z, McGoogan MJ (2020) Characteristics of and important lessons from the coronavirus disease 2019 (COVID-19) outbreak in China: Summary of a report of 72314 cases from the Chinese Center for Disease Control and Prevention. JAMA 323: 12391242. 
Citation: Franssen JMP, Kwee RM, Kruif MD, et al. (2021) Prediction of Mortality and Clinical Decisions Using Frailty Scores in Hospitalized Elderly with Covid-19. T Geriatr Med 3(1):49-54

5. Verity R, Okell LC, Dorigatti I, et al. (2020) Estimates of the severity of coronavirus disease 2019: A model-based analysis. Lancet Infect Dis 20: 669-677.

6. Hubbard RE, Peel NM, Samanta M, et al. (2017) Frailty status at admission to hospital predicts multiple adverse outcomes. Age Ageing 46: 801-806.

7. Fried LP, Tangen CM, Walston J, et al. (2001) Frailty in Older Adults: Evidence for a Phenotype. J Gerontol A Biol Sci Med Sci 56: 146-156.

8. Xue $Q$ (2011) The frailty syndrome: Definition and natural history. Clin Geriatr Med 27: 1-15.

9. https://www.nice.org.uk/guidance/ng159

10. Rockwood K, Song X, Macknight C, et al. (2005) A global clinical measure of fitness and frailty in elderly people. CMAJ 173: 489495.

11. Dent $E$, Kowal P, Hoogendijk EO (2016) Frailty measurement in research and clinical practice: A review. Eur J Intern Med 31: 3-10.

12. Bai HX, Hsieh B, Xiong Z, et al. (2020) Performance of radiologists in differentiating COVID-19 from viral pneumonia on chest CT. Radiology 10: 200823.

13. Charlson ME, Pompei P, Ales KL, et al. (1987) A new method of classifying prognostic comorbidity in longitudinal studies: Development and validation. J Chronic Dis 40: 373-383.

14. Xie X, Zhong Z, Zhao W, et al. (2020) Chest CT for Typical 2019nCoV Pneumonia: Relationship to Negative RT-PCR Testing. Radiology: 200343.

15. Kundi H, Wadhera RK, Strom JB, et al. (2019) Association of Frailty With 30-Day Outcomes for Acute Myocardial Infarction, Heart Failure, and Pneumonia Among Elderly Adults. JAMA Cardiol 4: 1084-1091.
16. Robert De Smet, Bea Mellaerts, Hannelore Vandewinckele, et al. (2020) Journal Pre-proof.

17. Ma HM, Yu RH, Woo J (2013) Recurrent hospitalisation with pneumonia is associated with higher 1-year mortality in frail older people. Intern Med J 43: 1210-1215.

18. Darvall JN, Bellomo R, Paul E, et al. (2019) Frailty in very old critically ill patients in Australia and New Zealand: A population-based cohort study. Med J Aust 211: 318-323.

19. Escudero-Acha P, Palomo Navarro $M$, Leizaola Irigoyen $O$, et al. (2019) Preliminary results of the ADENI-ICU trial: Analysis of decisions of refuse admission in intensive care units as a limitation of life support treatments; multi-center, prospective, observational study. Medicina Intensiva 43: 317-319.

20. Rodriguez-Morales A, Cardona-Ospina J, Gutiérrez-Ocampo E, et al. (2020) Clinical, laboratory and imaging features of COVID-19: A systematic review and meta-analysis. Travel Med Infect Dis 34: 101623.

21. Richardson S, Hirsch JS, Narasimhan M, et al. (2020) Presenting Characteristics, Comorbidities, and Outcomes Among $5700 \mathrm{~Pa}-$ tients Hospitalized With COVID-19 in the New York City Area. JAMA 323: 2052-2059.

22. Shahid Z, Kalayanamitra R, McClafferty B, et al. (2020) COVID-19 and Older Adults: What We Know. J Am Geriatr Soc 68: 926-929.

23. Rockwood K, Mitnitski A, Song X, et al. (2006) Long-term risks of death and institutionalization of elderly people in relation to deficit accumulation at age 70. J Am Geriatr Soc 54: 975-979.

24. Song X, Mitnitski A, Rockwood K (2010) Prevalence and 10-year outcomes of frailty in older adults in relation to deficit accumulation. J Am Geriatr Soc 58: 681-687.

25. Clegg A, Young J, Iliffe S, et al. (2013) Frailty in elderly people. Lancet 381: 752-762.

DOI: $10.36959 / 452 / 582$

Copyright: (C) 2021 Franssen JMP, et al. This is an open-access article distributed under the terms of the Creative Commons Attribution License, which permits unrestricted use, distribution, and reproduction in any medium, provided the original author and source are credited. 